\title{
LA MOBILITÉ SPATIO-TEMPORELLE DANS TARMAC DE NICOLAS DICKNER
}

\author{
Veronika ČERNÍKOVÁ \\ Université de Bohême du Sud, České Budějovice
}

\begin{abstract}
En): The present article deals with the role of the movement in the Quebec novel Apocalypse for beginners (Tarmac) published by Nicolas Dickner in 2011. Firstly, the movement made by the protagonists in space is observed. In some parts of the novel the travel is real, sometimes it's virtual but the movement is always performed between the periphery and the centre, between here and elsewhere. Subsequently, the attention is paid to the temporal dimension of travel and to the movement between the present, past and future. Finally, the article focuses on the permeability of the notions of space and time.
\end{abstract}

Keywords (En): space; time; permeability; mobility; Quebec novel; travel

Mots-clés (Fr) : espace ; temps ; perméabilité ; mobilité ; roman québécois ; voyages

L'œuvre romanesque de l'auteur québécois Nicolas Dickner représente un corpus relativement homogène de trois romans polyphoniques mettant en scène des personnages d'adolescents en quête d'identité. La narration de son premier roman, Nikolski (2005), alterne entre le « je » anonyme, Joyce et Noah, tous les trois étant demi-frères et demi-sœurs sans le savoir. Quoiqu'ils habitent tous Montréal, ils n'entrent en contact que par l'intermédiaire d'un livre mystérieux, dit «à troistêtes » car composé de trois fragments de livres anciens. Ces fragments, remarque Christine OTIS (2009), peuvent être associés aux protagonistes : la monographie sur les îles aux trésors à Noah qui fait des fouilles archéologiques sur l'île de Stevenson ; le traité sur les pirates des Caraïbes à Joyce qui descend d'une famille de pirates et trouve sa vocation dans la piraterie informatique ; la biographie d'un naufragé sur une île déserte au «je » anonyme qui est collectionneur de guides de voyage et de cartes géographiques tout en étant coincé dans sa librairie déserte. Chacun mène une sorte d'apprentissage tardif qui lui permet finalement de passer à l'âge adulte. Tarmac (2011), deuxième roman de Dickner, introduit l'histoire de deux adolescents, Hope et Michel alias Mickey, qui se rencontrent dans une petite ville québécoise à la fin de la guerre froide. Leur amitié d'une intensité exceptionnelle est interrompue par le départ de Hope à New York, d'où elle voyage à Seattle pour s'installer à Tokyo. Ce n'est qu'après dix ans de silence que Hope envoie son adresse à Mickey. Lui, jusque-là incapable de se lancer dans une relation amoureuse de longue durée, décide de partir à sa recherche. Le troisième roman, Six degrés de liberté (2015), représente une sorte d'amalgame des deux précédents. Semblablement à Nikolski, il y a trois protagonistes : la pirate informatique quadragénaire Jay et deux adolescents Eric et Lise. Christian DESMEULES (2015) et Anne-Marie BILODEAU (2015) observent que Jay n'est autre que Joyce, pirate informatique de Nikolski. Sa ligne narrative est de plus strictement séparée de celle des adolescents car le temps n'y passe pas à la même vitesse, comme le note Josée LAPOINTE (2015), et que Jay ne suit pas les activités des adolescents directement mais en ligne. Lise et Eric ont, eux, beaucoup de choses en commun avec Mickey 
et Hope. Ils habitent un petit village aux confins du Québec et leur amitié est rompue par le départ soudain d'Eric qui s'installe avec sa mère au Danemark. Pareillement à Tarmac, ce départ cause le partage de la trame narrative entre deux protagonistes. En outre, Nicolas Dickner lui-même souligne que, suivant l'exemple de ses romans précédents, il a placé au centre de l'intrigue de son dernier roman la géographie. D'après l'auteur, ce « roman du conteneur » (LAPOINTE, 2015) est « postgéographique » et «porte sur la négation du territoire » (DESLOGES, 2013). Tout en rêvant de rester ou de devenir voyageurs, les personnages de Six degrés de liberté incarnent davantage la figure du captif : Jay est condamnée pour vol d'identité et doit purger sa peine au service de la Gendarmerie royale du Canada ; Eric souffre d'une agoraphobie qui ne lui permet pas de quitter sa chambre; et Lise, pour retrouver Eric, réalise son voyage enfermée dans un conteneur réfrigérant pendant plusieurs mois et traverse les océans sans connaître les paysages, sans rencontrer âme qui vive (DESMEULES, 2015).

Dans tous ses romans, Nicolas Dickner introduit des personnages qui descendent de nomades comme Noah (Nikolski) ou Hope (Tarmac); qui rêvent de voyages comme le collectionneur de cartes (Nikolski); qui effectuent des voyages virtuels comme Hope et Mickey par l'intermédiaire de la télévision (Tarmac), ainsi que cybernétiques comme la pirate informatique Joyce/Jay (Nikolski et Six degrés de liberté) ou Eric, inventeur du programme de gestion de déplacement des conteneurs (Six degrés de liberté). Les voyages dans l'espace sont doublés de ceux dans le temps : Noah est étudiant en archéologie (Nikolski), Mickey est amateur d'archéologie et de science-fiction (Tarmac), le père dément de Lise est incapable de distinguer le présent du passé (Six degrés de liberté). Les déplacements des personnes sont enrichis de déplacements, parfois inexplicables, d'objets : l'étrange livre à trois têtes qui change de propriétaire un peu à son gré (Nikolski), des bâtiments qui disparaissent à Rivière-du-Loup pour apparaître à Tokyo (Tarmac), le conteneur mystérieux qui déplace Lise du Québec au Danemark (Six degrés de liberté). Comme les trois romans de Dickner sont polyphoniques, c'est aussi la narration qui se déplace entre les différentes voix narratives tout en suivant alternativement l'histoire de deux (Tarmac) ou de trois héros (Nikolski et Six degrés de liberté). Si les trois romans dickneriens placent le déplacement dans l'espace et le temps au centre de leur intérêt, c'est uniquement dans Tarmac que Dickner travaille également le déplacement de l'espace et du temps eux-mêmes. De ce fait, le texte qui suit proposera une réflexion non seulement sur le mouvement dans l'espace et dans le temps mais aussi sur la mobilité, la fluidité et la perméabilité de l'espace et du temps dans Tarmac ${ }^{l}$.

\section{Entre la périphérie et le centre}

Hope et Mickey, les protagonistes de Tarmac, sont tous les deux descendants de familles marquées par un déplacement forcé. Hope est issue d'une "famille d'origine très vaguement acadienne » (p. 18) déportée par les Britanniques en 1755 au Maryland et revenue en Nouvelle-Écosse sans se laisser assimiler. Ce long aller

\footnotetext{
${ }^{1}$ Tous les renvois à la pagination se rapportent à l'édition DicKNER Nicolas (2011), Tarmac, Montréal, Alto.
} 
et retour a probablement ravagé la santé psychique de la famille désormais obsédée par l'apocalypse. Le lien entre le nomadisme forcé et la psychose apocalyptique devenue héréditaire est explicité par le narrateur :

On pouvait d'ailleurs croire que l'obsession familiale pour l'apocalypse remontait à ce traumatisme géopolitique. N'était-il pas normal, voire inévitable, qu'une lignée d'agriculteurs déportés éprouvât certaines sensibilités à l'égard des agglomérations urbaines, des grandes catastrophes et du cours normal de l'histoire ? (p. 18)

Avec Ann Randall, la mère de Hope, la logique se renverse et c'est la psychose apocalyptique qui initie les déplacements familiaux. Une nuit de juillet 1989, en attendant la fin du monde, Ann décide de partir vers l'ouest. Elle croit gagner du temps « partant peut-être du principe que les fuseaux horaires allaient en décroissant » (p. 31). Avec sa fille, elles quittent Yarmouth, une toute petite ville située dans la région la plus méridionale de la Nouvelle-Écosse et du Canada, pour traverser l'ancien territoire acadien. Leur voyage est effectué sur le bord d'une vieille Lada, remplie jusqu' au plafond par des denrées alimentaires et une collection de bibles. Dès qu'elles traversent la frontière québécoise près du Témiscouata, le décor préapocalyptique est étoffé par «le souffle jaunâtre des incendies de forêt » (p. 33). C'est près de Rivière-du-Loup que le « cœur de camarade Lada claqu[e] brusquement » (p. 34) ce qui force Anne à s'y installer dans une ancienne animalerie L'Arche de Noéh. Un mardi matin du mois d'août 1990, Anne décide de déménager à Puerto Plata en République dominicaine, mais le voyage dans le Sud n'a pas lieu car Hope le refuse.

Pourtant, Hope se détermine à entreprendre son propre voyage apocalyptique au printemps 1991 quand sa date de fin du monde se voit confirmée par le prophète Hayao Kamajii alias Charles Smith. En quête de ce dernier, elle prend l'autobus pour aller à New York d'où elle continue à Seattle, toujours en autobus. Découvrant que Mekiddo, la compagnie du prophète, a entretemps déménagé à Tokyo, elle part finalement au Japon. Au cours de son voyage qui commence par la traversée du continent et finit par la traversée de l'Océan pacifique, Hope suit la trajectoire du Soleil sans s'écarter une seule fois de la direction de l'Ouest.

À Tokyo, Hope continue à poursuivre le prophète et sa compagnie qui changent de siège du jour au lendemain, ce qui rythme son séjour par des allers et retours incessants. Bien que présentés par le narrateur comme factuels, ses voyages se déroulent dans un espace virtuel. Au lieu de permettre au lecteur de suivre le mouvement de Hope à travers un Tokyo forcément fantastique, les toponymes l'amènent en Israël. Ainsi, Hope cherche les locaux de Mekiddo sur le boulevard Akko même si aucun boulevard de Tokyo ne porte ce nom. Akko est en effet une ville dans le nord d'Israël qui a joué un rôle important lors des croisades médiévales. Il n'est pas surprenant alors que Mekiddo ne s'y trouve pas. D'autres adresses de Mekiddo amènent Hope dans les arrondissements Nayot et Gilo. Merriam, amie et protectrice de Hope, précise que Nayot est un « arrondissement voisin » (p. 182). Néanmoins, il porte le nom d'un quartier de Jérusalem habité par les immigrés des États-Unis et du Canada. Gilo, par contre, devrait se trouver à l'autre bout de la ville mais en réalité, Gilo est une ancienne ville biblique et actuellement un autre quartier de Jérusalem. Le toponyme le plus paradoxal est celui de Jaffa où Merriam habite 
et travaille et où Hope est logé : «C'est un des rares bâtiments de la période Edo qui a survécu à la fois au tremblement de terre de 1923, aux bombardements de 1945 et à la vague d'urbanisme des années 60 » (p. 191). Pourtant, Jaffa porte le nom d'une vieille ville portuaire qui a été appelée d'après le fils de Noéh et qui constitue aujourd'hui un quartier de Tel-Aviv. Situé « en plein centre de la mer des Sargasses » (p. 192), tout comme le triangle des Bermudes, Jaffa est un bâtiment on ne peut plus fantastique. Nulle part et partout, il incarne la multidimensionnalité et la perméabilité de l'espace romanesque. Le lien étrange et inhabituel entre le Japon et Israël est d'ailleurs incarné par Merriam, elle-même née à Jérusalem d'une mère juive et d'un père japonais.

Mickey, de son côté, descend d'une famille de bâtisseurs de mondes qui, débarquée de Hollande au milieu du $19^{\mathrm{e}}$ siècle, s'est installée au New Jersey. Pareillement aux Randall, les Bauermann subissent un traumatisme suite à un déplacement forcé quand la famille a été « écartée du marché régional par la mafia » (p. 48). «L'exode des Bauermann $»^{2}$ (p. 48) s'est produit en 1953 : ils sont montés en Nouvelle-Angleterre, qu'ils ont traversée jusqu'au Canada et se sont arrêtés à Rivière-du-Loup. Ils devaient laisser le soin de bâtir New York à d'autres tout en réajustant leurs ambitions car " les dépotoirs canadiens étaient moins vastes, les usines plus petites » (p. 49). Sinon, les parents de Mickey sont des sédentaires comme l'indique leur nom dont l'origine germanique fait penser aux paysans ou fermiers.

Mickey non plus ne paraît pas très enclin aux voyages. Son déménagement à Montréal est passé sous silence et son aller et retour de Montréal à Rivière-du-Loup à l'occasion de l'enterrement d'Ann Randall ne lui est pas trop agréable : «Et, une fois de plus, je me tapais l'interminable trajet vers ma ville natale : cinq heures de route rectiligne, plusieurs litres de thé glacé et une pause élimination à mi-chemin. » (p. 238) D'autant plus surprenante peut paraître sa décision finale de rejoindre Hope à Tokyo. Il est à noter qu'avant d'arriver à Tokyo, il doit faire trois escales : à Acapulco (Mexique), à San-Diego (Californie) et à Honolulu (Hawaii). La première escale le fera traverser le continent du nord-est au sud-ouest. La troisième indique l'arrêt à mi-chemin de la traversée de l'Océan pacifique, tout près de l'antiméridien et de la ligne de changement de date. Or, ce voyage reste à réaliser car le roman s'achève au moment de l'embarquement.

Les déplacements qui caractérisent l'histoire de Tarmac entraînent l'oscillation du récit entre la périphérie et le centre. Dickner situe l'acte narratif à Rivière-duLoup dont il fait la ville natale du narrateur Mickey. Il s'agit d'un véritable bout du monde, périphérie à la fois américaine, canadienne et québécoise. C'est là que se déroule l'action de la première partie du livre. Après le départ de Hope qui se produit au beau milieu du récit (au chapitre 49 sur un nombre total de 97 chapitres) et qui coupe le roman en deux parties, l'action est répartie entre plusieurs lieux d'après le séjour de Hope : cinq chapitres se déroulent à New York, sept à Seattle et vingt à

\footnotetext{
${ }^{2}$ Deux autres exodes sont mentionnés dans le roman sans toucher directement les protagonistes. D'une part, il s'agit de l'histoire des boat people, six ou sept familles de Laotiens qui ont immigré à Rivièredu-Loup à la fin des années 1970. D'autre part, la télévision diffuse les images du rapatriement de quinze mille Juifs éthiopiens qui, d'après les mots d'un journaliste, «terminent un voyage de trois mille ans !» (p. 229).
} 
Tokyo. Mickey reste ancré à Rivière-du-Loup jusqu'au chapitre 86, ensuite l'acte narratif se déplace à Montréal (chapitres 87-97) même si interrompu par son retour temporaire à Rivière-du-Loup (chap. 88-90). Plusieurs coups de fil relient la périphérie de Mickey (Rivière-du-Loup) aux centres de Hope (New York et Seattle). Le dernier appel que Hope voulait effectuer de Tokyo n'est jamais réalisé. Jusqu'à l'avant dernier chapitre où Mickey reçoit la lettre indiquant l'adresse de Hope, le récit suit deux lignes strictement séparées ce qui cause non seulement la susdite oscillation entre la périphérie de Mickey et le centre de Hope mais aussi entre deux types de narration. En effet, à partir du chapitre 49, le narrateur homodiégétique alterne avec le narrateur hétérodiégétique sans que l'auteur recoure nécessairement au changement d'instance narrative : il paraît que c'est Mickey adulte qui raconte les aventures de son adolescence ainsi que celles de Hope dont il ne savait rien à l'époque. Ce changement de stratégie narrative souligne non seulement la différence entre la première et la seconde partie du livre mais surtout met en relief l'écart entre la périphérie racontée à la première personne et le centre narré à la troisième personne. La périphérie incarne ce qui est connu, proche et compréhensible ; le centre ce qui est inconnu, lointain et incompréhensible. Dickner l'explicite dans une interview accordée à Josianne DESLOGES : «dans Tarmac, il était question de ce que c'est de vivre dans une époque préapocalyptique dans une petite ville, par rapport à New York ou Tokyo. » (2013)

La différence d'ordre ontologique entre la périphérie et le centre entraîne aussi un différent traitement de l'espace. Incarnant la périphérie, Rivière-du-Loup est présentée d'une manière réaliste mais assez dépressive comme une ville morte, « convenable pour attendre la fin du monde » (p. 34). Ses établissements sont soit abandonnés comme l'épicerie ou le stade municipal où il n'y a personne en vue, soit fermés : la Polyvalente pour une journée, le cinéma Princesse pour une semaine, le cinéparc depuis des années. La piscine municipale extérieure, " menacée de fermeture définitive à chaque printemps » (p. 113), finit par disparaître après le départ de Hope pour Tokyo. Le même sort attend un peu plus tard aussi le stade municipal, détruit lors d'un incendie. Cette destruction est très mal vue par Mickey, amateur des temps anciens. Pour lui, la piscine et le stade représentent deux points de repère cruciaux, l'une à cause de sa puissance purificatrice et l'autre en tant que lieu de sa première rencontre avec Hope. Dès l'incipit, Rivière-du-Loup est associée à la destruction. Après avoir constaté que la piscine extérieure est encore une fois fermée, Mickey se rend au stade désert où il rencontre Hope. Celle-ci imagine ce qui arriverait si la bombe d'Hiroshima explosait au-dessus de la ville : « Le centre commercial volerait en miettes, les bungalows seraient soufflés, les voitures projetées comme des boîtes de carton, les lampadaires s'allongeraient au sol. » (p. 13) Comme si cette destruction imaginée par Hope anticipait la destruction de la Rivière-du-Loup de l'adolescence de Mickey qui aura lieu à la fin du roman. Or, la destruction n'est que prétexte à la construction. Celle de la piscine et du stade entraîne la construction d'une maison de jeunes et de trente condominiums ce qui amène Mickey à la réflexion que « le bungalow reste la principale arme de destruction massive inventée durant la guerre froide » (p. 234).

Contrairement à Rivière-du-Loup, Tokyo est présenté comme une ville fantastique. Outre les toponymes reliant la ville japonaise à la géographie 
israélienne, l'élément fantastique est mis en évidence par les improbables et incessantes mutations de cette ville à " géographie variable », d'après l'expression d'Anne-Josée CAMERON (2010). Vingt-huit fois, Hope part à la recherche des locaux de Mekiddo et vingt-huit fois, elle arrive avec « quelques heures - voire quelques jours - de retard sur la réalité » (p. 199). Elle ne tombe que «sur des édifices abandonnés ou à vendre, des terrains vagues, des stationnements improvisés, des chantiers de démolition ou, plus rarement, des bâtiments flambant neufs, apparus au cours de la nuit comme des champignons » (p. 199). La multinationale Mekiddo accuse la capacité inouïe de déménager en 72 heures quelques centaines d'employés, de chaises et de bureaux. Les déménagements de la compagnie ne font que souligner la caractéristique principale de la ville qui consiste dans sa mutation constante. Merriam le dit clairement : « rien ne restait en place très longtemps et le paysage se métamorphosait à une vitesse stupéfiante. On pouvait emprunter la même rue tous les matins et, du jour au lendemain, ne plus rien reconnaître. » (p. 186) La métaphore de la vitesse permet à l'auteur de montrer qu'un habitant d'une petite ville somnolente ne peut pas vraiment comprendre une métropole mondiale grouillante et en pleine expansion ce qui, encore une fois, augmente l'écart entre ici et ailleurs, c'est-à-dire entre la périphérie et le centre, mais aussi entre l'Occident et l'Orient. Pour Mickey et Hope, Tokyo est une ville à topographie fantaisiste, une ville étrange, bizarre, incompréhensible où même les « rues vont dans n'importe quel sens » (p. 191).

Néanmoins, Tokyo et Rivière-du-Loup ont une chose en commun : la vie y est rythmée par les destructions et les constructions. Ce lien est mis en évidence par deux bâtiments, la piscine municipale et le stade de baseball, dont la présence dans les deux villes frôle le fantastique. Si la piscine municipale disparait de Rivière-duLoup, une flambant neuve vient d'apparaître à Tokyo, à l'endroit où « devaient indubitablement s'élever les bureaux de Mekiddo» (p. 172). Deux semaines après la piscine, le stade de baseball de Rivière-du-Loup disparait dans l'incendie comme s'il voulait faire place à celui de Tokyo. Tout comme la piscine de Tokyo, le stade se dresse à la place des bureaux de Mekiddo et il est « flambant neuf, apparemment érigé au cours de la nuit» (p. 210). Les deux stades sont d'ailleurs les lieux de rencontres cruciales : au stade de Rivière-du-Loup, Mickey rencontre pour la première fois Hope ; au stade de Tokyo, Hope rencontre le prophète Hayao Kamajii. L'effet fantastique de la dis-/apparition du stade est renforcé par la disparition inattendue de Hayao Kamajii : entré «dans une toilette chimique abandonnée sur place après la construction du stade » (p. 222), il n'en est jamais sorti. ${ }^{3}$ Les de/constructions fantastiques des piscines municipales et des stades de baseball créent l'impression de la perméabilité de l'espace où n'importe quoi et n'importe qui peut à volonté disparaître ou apparaître.

Cette perméabilité spatiale entre la périphérie et le centre fonctionne aussi dans le sens inverse. À Rivière-du-Loup, les protagonistes consomment presque

\footnotetext{
${ }^{3}$ Dans son étude «Tarmac ou l'apocalypse selon Nicolas Dickner », Petr KYLOUSEK éclaircit l'origine du nom du prophète : «Kamaji (sans le $i$ double) est le personnage de l'araignée (donc Spider-Man) du dessin animé culte Sen to Chihiro no kamikakushi (Le voyage de Chihiro, 2001) de Hayao Miyzaki. » (2015 : 35-36) La référence au super-héros souligne l'ambiance fantastique de Tokyo tout en insinuant l'explication de la disparition mystérieuse du prophète.
} 
uniquement les ramens Captain Mofuku, soupe japonaise à l'emballage emblématique représentant « un cosmonaute rosé et jaune flotta[ant] autour d'une planète de nouilles » (p. 70). En outre, Mickey découvre l'existence d'une autre « récente invasion asiatique », le tofu :

Pour le moment, il s'agissait d'une denrée exotique et peu ragoûtante. Dans quelques années, elle serait parfaitement intégrée à notre diète, banale comme le Nutella ou la bombe H. Nous serions, au terme de cette Grande Guerre du Tofu, un tout petit peu plus asiatiques - mais personne ne s'en apercevrait. (p. 90)

Quelques mois plus tard, sa mère non seulement cuisine au tofu mais aussi pratique la méditation zen et achète des bouddhas et des bonsaïs. Mickey finit d'ailleurs par constater que Rivière-du-Loup ressemble à une aquarelle japonaise. Est-ce le hasard que cela se passe quand ses pas le mènent à l'endroit où se dressait le stade municipal quelques semaines plus tôt ? Même si le réseau d'échos entre Rivière-du-Loup et Tokyo peut être interprété comme un effet de globalisation culturelle $^{4}$, nous insistons sur son potentiel fantastique. La perméabilité issue de l'agencement spatial symétrique avec deux espaces dotés de « deux stades de baseball, deux bars aux noms bibliques (le Jaffa et l'Ophir), deux 'refuges' habitables, la maison secrète de Merriam et le bunker» (SIMARD-HoudE, 2009) et reliés par « un étrange jeu de miroir », actualise l'univers « distordu », fantastique et perméable des fictions de l'écrivain japonais (sic !) Haruki Murakami (CAMERON, 2010). ${ }^{5}$ De ce fait, la perméabilité spatiale dépasse les limites de la narration car elle permet à Nicolas Dickner, auteur québécois relativement inconnu, d'entrer en dialogue avec Haruki Murakami, auteur japonais reconnu actuellement à travers le monde entier.

\section{Entre l'archéologie et la science-fiction}

Tout en étant mis en évidence par de nombreux éléments, tels les voyages ou la topographie fantastique, l'espace de Tarmac est loin de représenter la catégorie narrative la plus travaillée par Dickner. Dès l'épigraphe, l'auteur attire l'attention du lecteur sur le temps : «The future ain't what it used to be ». Le paratexte donne l'auteur de la citation, le joueur de baseball Yogi BERRA qui en a offert l'explication dans son livre The Yogi Book: I really didn't say everything I said! : « I just meant that times are different. Not necessarily better or worse. Just different. » (1998: 1189) La citation de Berra implique à la fois le futur (L'avenir), le présent (n'est plus) et le passé (ce qu'il était), l'un cédant le pas à l'autre, l'un se transformant en l'autre car il n'est pas possible de penser le présent sans le passé par qui il est déterminé ni sans l'avenir qu'il détermine. Posant le problème de l'inconstance et de la perméabilité des notions du passé, du présent et de l'avenir, l'épigraphe introduit un

\footnotetext{
${ }^{4}$ D'après Mélodie SIMARD-HoudE, le roman «met bien en évidence 'l'homogénéité hétérogène' qui caractérise la culture à l'ère contemporaine : partout la même diversité, le même mélange culturel. » (2009)

${ }^{5}$ Notons que le recueil de nouvelles de Murakami, L'éléphant s'évapore, figure sur la liste des 24 livres faisant partie de la bibliothèque idéale de Nicolas Dickner (« La bibliothèque idéale de Nicolas Dickner », 2015).
} 
leitmotiv traversant l'œuvre entière. Néanmoins, sous cette citation populaire, nous pouvons en repérer une autre, plus subtile, écrite par le poète Paul VALERY en 1937 dans son essai Notre destin et les lettres : «L'avenir est comme le reste : il n'est plus ce qu'il était. J'entends par là que nous ne savons plus penser à lui avec quelque confiance dans nos inductions. Nous avons perdu nos moyens traditionnels d'y penser et de prévoir : c'est le pathétique de notre état. » (1945: 156). L'idée de Valéry implique non seulement la mobilité du temps mais aussi notre incapacité de prévoir les changements. Et c'est cette incapacité qui est thématisée par le roman. En outre, la double citation indique la nature complexe du roman et de la réalité dont il traite : il ne faut pas se fier aux apparences.

La perméabilité des notions temporelles est explicitement remarquée par le narrateur qui se rend compte que ce qui relevait de la science-fiction pour nos ancêtres devient pour nous tout à fait banal :

Pour le citoyen moyen de 1945, la bombe atomique venait du futur, au même titre que les extraterrestres de La guêtre des mondes. [...] Les enfants de l'après-guerre avaient vécu l'arrivée du Boeing 747, du LSD et de la bombe H, et lorsque ma génération était entrée en scène, les missiles intercontinentaux de 100 kilotonnes appartenaient déjà à l'histoire ancienne. Ils étaient comme le four à micro-ondes, les ramens Captain Mofuku à saveur de poulet ou la télévision satellite : un rouage de la réalité. (p. 63-64)

Mickey est d'ailleurs obsédé à la fois par le passé et l'avenir. Sa bibliothèque possède deux sections dont il souligne la « complémentarité naturelle» (p. 45) : la science-fiction occupant la partie haute de la bibliothèque et l'archéologie située symboliquement au ras du plancher de son sous-sol. Il suit avec fascination un reportage sur les fouilles de Pompéi qui laisse Hope, avide de nouvelles, indifférente. Il est un grand lecteur d'Isaac Asimov, des romans de science-fiction en général et des bandes dessinées sci-fi comme Godzilla Roi des Monstres Affronte Capitaine America. C'est dans cette BD qu'il découvre la «publicité de lunettes Amazing X-Ray Vision capables de transpercer la matière (incluant les vêtements féminins) » (p. 52) dont Hope se moque ouvertement. Il est touché en particulier par ce qu'il appelle les « ruines virtuelles » (p. 258) offrant « le dosage idéal d'archéologie et de science-fiction » : les bâtiments et les constructions en béton qui fonctionnent encore mais dont on annonce « la mort imminente depuis des années » (p. 257). Ses lieux préférés sont le stade de baseball et la piscine municipale, « une vieille chose craquelée, bâtie après la guerre (on ne savait plus trop laquelle) et menacée de fermeture définitive à chaque printemps » (p. 113). Mickey éprouve « un plaisir proprement archéologique à nager dans ces structures vétustés » qui lui rappellent « les thermes de Rome, quelques mois avant la chute de l'Empire » (p. 113). Un autre lieu qui le fascine est l'aéroport Mirabel dont il s'imagine l'abandon :

Combien de temps faudrait-il avant que le chiendent ne s'infiltre dans les joints de ce béton impeccable ? Que le tarmac ne soit perforé par les touffes de foin, la hart rouge, les saules et les aulnes ? [...] Le terminal était désert, à la fois paisible et déprimant. Il n'y manquait qu'une poignée de morts-vivants. (p. 258)

Impliqué même dans le titre du roman, le béton joue un rôle important dans la vie de Mickey. Il descend d'une famille exploitant, à l'aube de la Seconde Guerre 
mondiale, l'une des plus grosses cimenteries de la région de New Jersey. De plus, le béton et le ciment incarnent la base de notre civilisation : inventés à la période de l'Empire Romain, ils assurent la pérennité des bâtiments antiques aussi bien que contemporains. Il est même possible de trouver un parallèle entre la cimenterie familiale et la ville de Pompéi, les deux couverts d'une fine couche de poussière. Il n'est pas surprenant que quand il doit rédiger son devoir de composition sur le thème « À quoi ressemblera le monde du futur » (p. 51), Mickey décide d'écrire sur le béton: « Je prédisais une architecture révolutionnaire, basée sur de toutes nouvelles variétés d'additifs. (Dans le registre science-fictionnel, le mot 'additif' me semblait plutôt convaincant.) » (p. 57) Le béton est un matériau qui relie le passé à l'avenir, qui relève de l'archéologie et de la science-fiction.

Hope de son côté passe tout son temps libre à suivre l'actualité à la télévision et dans les journaux : fin de la guerre froide, chute du mur de Berlin, incident de Tchernobyl, attentat sur René Moawad, invasion du Koweït suivi du bombardement de Bagdad et beaucoup d'autres événements. "Toujours en phase avec l'actualité », Hope annonce dans son devoir de composition, au titre révélateur «boum », « la chute du régime Soviétique et la fin de la guerre froide d'ici les deux prochaines années, et elle ajout[e] que bientôt nous cesser[ons] de craindre la bombe atomique. Désormais, nous redouter[ons] l'obsolescence industrielle de l'URSS - comme, d'ailleurs, l' [a] démontré l'incendie de Tchernobyl. » (p. 57) Sa vision de l'avenir est déterminée par le développement de la situation actuelle. De plus, Hope « est une jeune fille assoiffée de science »: " astronomie, génétique, chimie, tout l'intéress[e] » (p. 27). Or, l'obsession majeure de Hope est celle de la fin du monde qui constitue son héritage familial. Chaque membre de la famille annonce une date différente de fin du monde accompagnée d'une vision apocalyptique en trois dimensions : Harry Randall Truman, le premier cas attesté, présage le retour de Moïse à bord d'une baleinière incandescente à l'automne 1835, la grand-mère de Hope attend vainement un orage de feu en été 1966 et son grand-père annonce l'Armageddon pour le 12 juin 1977. La mère de Hope néanmoins ne reçoit qu'une vision incomplète, privée de détails. Elle sait seulement que la fin du monde se produira en été 1989. Hope n'aura plus de vision du tout, elle trouve la date de la fin du monde en jouant aux dés : le 17 juillet 2001. Pour le malheur des Randall, la planète persiste « à tourner telle une mauvaise blague » (p. 20). La fin du monde tant attendue n'a pas lieu : ce qui constituait l'avenir en quelque sorte rassurant appartient désormais au passé et les membres de la famille de Hope se perdent dans un chaos insupportable. Assez paradoxalement, la fin du monde connote l'avenir aussi bien que le passé et le présent. Vu qu'il y a toujours de nouvelles fins du monde annoncées par une grande quantité de prophètes, le présent peut être perçu comme le moment entre les fins du monde qui n'ont pas eu lieu dans le passé et celles qui pourraient avoir lieu dans l'avenir. De plus, avec le nombre de prophètes, la représentation de la fin du monde dans le roman prend des allures des plus variées : du Déluge biblique à l'invasion des morts-vivants en passant par l'explosion de la bombe atomique.

La bombe représente d'ailleurs une autre obsession importante de Hope. Elle ne s'imagine pourtant pas les bombes modernes puisque leur puissance, explique-telle, «dépasse l'imagination »: « Prends un missile balistique banal, environ 500 
kilotonnes. L'explosion pourrait catapulter un bout de plaque tectonique en orbite. Le cerveau humain ne peut pas comprendre ça. » (p. 12-13) La Bombe de Hope, c'est Little boy car il est «plus facile à mesurer » (p. 13). Ainsi, c'est la bombe d'Hiroshima qu'elle convertit en citrons et à laquelle elle pense même en regardant des films de zombies avec Mickey. La toute première phrase que Hope adresse à Mickey est : «La nuit dernière, j' ai rêvé de la bombe d'Hiroshima » (p. 12). Ce qui suit est un véritable exposé pédagogique, accompagné de chiffres précis et de démonstrations convaincantes :

Si ça explosait au-dessus de nos têtes, à environ 600 mètres d'altitude — comme à Hiroshima - alors l'onde de choc raserait la ville sur un rayon de 1,5 kilomètre. Ça donne une surface d'environ 7 kilomètres carrés. L'équivalent de... [...]... 2500 terrains de baseball.

Elle cessa un instant de déchirer l'emballage de Cracker Jack pour englober les environs d'un élégant geste pédagogique.

- Le centre commercial volerait en miettes, les bungalows seraient soufflés, les voitures projetées comme des boîtes de carton, les lampadaires s'allongeraient au sol. Et ça, c'est seulement l'onde de choc. Ensuite il y a la radiation thermique. Tout serait réduit en cendres sur des dizaines de kilomètres carrés - beaucoup, beaucoup de terrains de baseball ! À proximité de la bombe, la chaleur dépasserait la température à la surface du Soleil. Le métal entrerait en fusion. Le sable formerait des petites billes de verre.

Elle avait terminé l'opération déchiquetage, soupesait le tas de confettis dans sa paume.

- Et sais-tu ce qui nous arriverait, deux pauvres minuscules primates composés à $60 \%$ d'eau?

Elle renversa tout doucement sa main et la brise emporta sa poignée de confettis en direction du champ gauche.

— Nous serions vaporisés en trois millièmes de seconde. (p. 13-14)

De plus, cette première rencontre des adolescents se déroule sur un terrain de baseball et Hope a sur la tête une casquette des Mets de New York, le club de l'auteur de l'épigraphe Yogi Berra. L'avenir n'est pas ce qu'il était et pour la génération de Hope et de Mickey, l'avenir ne peut pas être pensé sans la bombe atomique. Mais on n'est pas capable d'imaginer l'avenir sans avoir recours à notre expérience et à nos connaissances. En imaginant l'avenir, on actualise le présent et le passé. Ainsi, le grand-père de Mickey s'imagine la bombe d'Hiroshima comme une « montagne de ces bâtonnets cartonnés que l'on utilisait dans les carrières de gypse », incapable de « comprendre en quoi la bombe d'Hiroshima différait de la bonne vieille dynamite » (p. 64). C'est pourquoi la bombe qui hante les adolescents de la guerre froide finissante est bien celle d'Hiroshima.

Non seulement Hope parle souvent de la bombe atomique, non seulement elle reproduit l'explosion à l'aide des crackers, non seulement elle observe tout ce qui peut faire penser à une explosion y compris le champignon de vapeur qui s'élève vers le plafond quand elle se prépare son ramen préféré, elle est une bombe atomique. Elle «bombarde d'informations » (p. 101) sa mère et elle organise des « raids » à Tokyo ayant pour but de trouver le prophète indiquant la même date de fin du monde qu'elle. En l'observant endormie, Mickey mène cette réflexion : « Le cerveau humain consommait prétendument le cinquième de l'énergie produite par le corps, mais celui de Hope en flambait visiblement davantage - et tandis qu'elle respirait tout doucement, les yeux clos, j'imaginais son cortex fissionner en silence des noisettes d'uranium 235 » (p. 46-7), matériau fissile à partir duquel on fabriquait 
les premières bombes nucléaires. Dans le couple, c'est elle qui agit la première, qui initie leurs activités. Elle est une émettrice de signaux reçus par Mickey. Elle dégage « un champ magnétique - la radiation de ses 195 points de Q.I. » (p. 14) et son discours sur la bombe d'Hiroshima produit une « onde de choc» (p. 14) qui endommage Mickey. Pour utiliser la métaphore du jeu de baseball, elle est un lanceur tandis que Mickey est un receveur. Cette binarité se manifeste également au niveau de la narration. Hope agit. Par son arrivée et son départ, elle met la vie de Mickey sens dessus dessous. Mickey subit. Tout ce qu'il fait, il le fait avec Hope. Il absorbe les aventures de Hope et persiste à les raconter même après son départ.

La binarité du couple est donnée aussi par leurs attributs, la bombe et le béton, qui correspondent à deux principes qui régissent le monde, la destruction et la construction. La famille de Hope souffrant de psychose apocalyptique est prédestinée à attendre la fin du monde et Hope elle-même affirme : « Je suis Shiva, le destructeur des mondes » (p. 111). Mickey déclare par rapport à sa famille : « Nous étions des bâtisseurs de mondes » (p. 49). Néanmoins, ces deux personnages et avec eux ces deux principes sont plus complémentaires qu'opposés. Le béton figurant la construction fait à plusieurs reprises référence à la destruction, pensons à la chute du mur de Berlin suivie à la télé par Hope et Mickey, aux destructions de la piscine municipale, du terrain de baseball et de l'aéroport Mirabel observées ou imaginées par Mickey. De l'autre côté, la bombe d'Hiroshima annonce le «début d'une ère nouvelle. Des maisons chauffées à l'énergie atomique. Des voitures au plutonium. Une source d'énergie illimitée. » (p. 59) Tout en étant le principal agent de la destruction, elle entraîne aussi la construction. Parallèlement, si Mickey est fasciné par l'archéologie et la science-fiction, il suit les événements mondiaux avec Hope, bien que ce ne soit jamais de son initiative et qu'il en soit plutôt désintéressé après son départ. Et à l'inverse, Hope regarde avec Mickey le reportage sur Pompéi tout en revendiquant l'actualité et le film de zombies ainsi qu'en cherchant des analogies avec Hiroshima. En effet, jusqu'à leur séparation, Mickey et Hope font tout ensemble, ils jouent dans la même équipe et puisqu'ils sont lanceur et receveur, ils jouent en défense. Ensemble, ils font face au passé et à l'avenir. D'une part, ils veulent échapper à la tradition de leurs familles. Mickey d'une manière consciente quand il refuse de continuer la cimenterie familiale et de procréer des enfants. Hope d'une manière inconsciente, car elle est la seule de la famille des Randall qui n'a pas eu de vision de la fin du monde mais qui en a obtenu la date par un coup de dés. $\mathrm{Si}$ les femmes de la famille reçoivent leur vision au moment de la première menstruation, Hope représente jusqu'à ses 29 ans une énigme médicale. Elle a ses premières règles le jour où sa fin du monde n'a pas eu lieu. Ou, peut-être, s'agit-il d'une fin du monde tout à fait symbolique et tout à fait personnelle, qui correspond à la fin symbolique et surtout tardive de l'adolescence. Et tout comme le présent qui est déterminé par le passé mais indéterminé par rapport à l'avenir, le roman luimême reste ouvert. L'auteur abandonne Mickey dans l'avion pour Tokyo où vit Hope sans préciser « la suite des choses » indiquée par le titre du dernier chapitre. Même si le ton de la dernière phrase est plus qu'optimiste : «Tout allait mieux depuis que la fin du monde était derrière nous. » (p. 259), il est possible de s'inquiéter par le numéro du vol 1707 qui correspond à la date de la fin du monde trouvée par Hope, le 17 juillet 2001. Si le 17 juillet a indiqué la fin de l'adolescence 
de Hope, le vol 1707 peut aussi bien indiquer la fin de l'adolescence de Mickey. Leur rencontre, insinuée par la stratégie narrative, fait penser au début d'une ère nouvelle, celle de la maturité symbolisée par la réunion du couple.

\section{Entre l'espace et le temps}

La perméabilité qui existe entre la périphérie et le centre ainsi qu'entre le passé, le présent et l'avenir fonctionne également entre l'espace et le temps, ce qui souligne l'interdépendance de ces deux catégories narratives. Le lien entre l'espace et le temps est mis en évidence notamment par le thème dicknerien par excellence : les voyages. Outre les voyages factuels réalisés par les protagonistes (voir ci-dessus), la narration est rythmée par les déplacements virtuels que les héros effectuent par l'intermédiaire des journaux et de la télévision. Il s'agit de brèves excursions virtuelles dans les centres des événements mondiaux. Non seulement Hope et Mickey passent tout leur temps libre à suivre les nouvelles, mais tous les événements importants de leur vie privée sont placés dans le contexte des événements de la politique mondiale, notamment des conflits militaires, et cela dès la première phrase du roman : "Août 1989 - Ronald Reagan avait quitté la Maison-Blanche, la guerre froide tirait à sa fin et la piscine municipale extérieure était (encore une fois) fermée. » (p. 11) Hope mémorise ses « premiers verbes irréguliers lorsque se produi[t] l'incident de Tchernobyl » (p. 28) et la condition de sa mère l'intéresse moins que la fusion à froid. Ensemble, Hope et Mickey célèbrent les dix-huit ans de Hope trois semaines avant le bombardement de Bagdad. Et finalement, l'atrium de la polyvalente ne laisse pas deviner que le mur de Berlin vient de tomber, puisqu'il est « à l'abri de l'histoire » (p. 58). Ce mouvement perpétuel et assez frénétique entre la périphérie et le centre réfère à l'Histoire tout en reliant le niveau individuel au niveau collectif. Ainsi, la vie quotidienne des protagonistes se déroule sur un fond historique spatialisé.

Un autre voyage virtuel à travers l'espace et le temps a lieu au Japon. Après sa première tentative de trouver Hayo Kamajii, Hope finit fatiguée à Jaffa's, bar où travaille Merriam. Le matin, elle se réveille dans une maison qui fait penser à « un véritable musée d'architecture traditionnelle japonaise » ou à un décor de cinéma « où le sens du réalisme aurait été poussé à l'extrême par un scénographe maniaque. » (p. 176) Hope éprouve un vertige quand elle découvre sur le mur un calendrier jauni, ouvert sur le mois d'août 1945. Pour un moment, elle croit qu'elle a «vraiment été catapultée dans le passé » (p. 177). Plus tard, Merriam lui explique que c'est la maison d'enfance de son patron et qu'il l'a fait déménager de Kokura dans les années 50. Tout en s'étant endormie dans le bar de Tokyo, Hope se réveille dans une ancienne maison de Kokura, ville qui a été « désignée comme la seconde cible au moment du lancement de la bombe atomique sur Hiroshima et comme la première cible au moment de Nagasaki », précise Kyloušek dans son étude sur l'ambiance apocalyptique de Tarmac, tout en ajoutant que « Kokura est une ville doublement miraculée, sauvée le jour de Nagasaki, par les pluies diluviennes qui obstruaient la visibilité requise pour le lancement. » (KYLOUŠEK, 2015 : 38) De plus, la maison de Kokura se trouve sur le toit du bâtiment Jaffa, un autre « survivant » miraculeux, et elle n'est accessible que par un escalier secret qui la 
relie directement au bar. Outre leur histoire similaire et l'escalier secret, les deux édifices partagent la présence de Merriam, mi-japonaise mi-juive, qui habite l'un et travaille dans l'autre. Et c'est précisément là-bas que Hope décide de s'installer. Par un réseau de références à l'Histoire de l'humanité, ce voyage virtuel qui se répète chaque jour relie le niveau individuel au niveau collectif.

En outre, l'aspect temporel des voyages est à plusieurs reprises explicitement commenté par les personnages. Hope explique que les douze années dont la sonde Voyager 2 avait besoin pour atteindre Neptune démontrent "l'immensité de l'univers et la minusculosité de notre planète » (p. 35). Lors de sa poursuite du prophète, Hope arrive avec «quelques heures - voire quelques jours - de retard » (p. 199) ce qui cause que « Hayao Kamajii rest[e] introuvable dans l'espace et dans le temps » (p. 200). Une fois, la distance de son déplacement est mesurée par une unité incarnant l'alliance de l'espace et du temps : " Hope débarqua au dixneuvième arrêt, à des années-lumière du centre-ville. » (p. 209) Le rapport entre la distance et la vitesse est d'ailleurs explicité par Merriam quand elle explique le phénomène de la «distance perçue » (p. 224) à Hope qui, après avoir marché « 20 kilomètres en pleine canicule » (p. 226), réalise que tout devait paraître considérablement plus éloigné avant l'invention de l'automobile. Merriam ne s'attarde pourtant pas sur la différence entre les moyens de transport mais entre les voyageurs. Elle prend l'exemple de la Palestine où « un cavalier pouvait parcourir jusqu'à 65 kilomètres par jour. Un piéton isolé, environ 40. Une armée bien disciplinée dépassait rarement les 30 kilomètres » (p. 224-5). La vitesse moyenne d'une famille «tombait sous la barre des 15 kilomètres par jour » (p. 225). Un autre facteur qui influence la vitesse des déplacements est leur urgence car si on fuit une menace, "l'armée du pharaon, une épidémie de morts-vivants ou la très sainte colère de Yahvé » (p. 225), alors cela change tout.

L'exemple le plus frappant de la mise en évidence de l'aspect temporel d'un voyage est représenté par les vols reliant l'Amérique au Japon. Hope traverse l'Océan pacifique à bord de l'avion qui relie directement Seattle à Tokyo. La descente finale commence à $15 \mathrm{~h} 32$, à la même heure où a commencé le décollage. En traversant l'antiméridien situé au milieu de l'Océan pacifique, les voyageurs traversent aussi la ligne de changement de date ce qui les fait « escamoter » vingtquatre heures. Hope est d'ailleurs «fascinée à l'idée qu'une journée complète se fût tout bonnement évaporée dans l'espace » (p. 167). De plus, le vol transpacifique est effectué dans le sens inverse de la rotation de la Terre, c'est-à-dire contre l'espace et contre le temps. L'un fait parvenir les voyageurs à l'extrême orient bien qu'ils poursuivent la direction de l'ouest ; l'autre les fait perdre une journée entière. Le mouvement dans le sens inverse actualise la métaphore du baseball, jeu où les joueurs se déplacent dans le sens inverse des aiguilles d'une montre. Attiré par le modèle perecquien ${ }^{6}$, Dickner paraît donner à la métaphore du baseball un rôle

\footnotetext{
${ }^{6}$ À la question «Quel est le livre qui vous a marqué, qui a changé votre vie ? » Dickner répond clairement : «Plusieurs livres m'ont marqué, mais La vie mode d'emploi occupe sans doute une place à part. J'ai toujours été estomaqué par l'ampleur, par l'ambition folle de ce romans - avec un s-, mais aussi par la finesse de Georges Perec, qui, non content de s'imposer des contraintes prodigieusement complexes, parvient à les dissimuler aux yeux des lecteurs. »(Cusson, 2015). Pour l'admiration que Dickner voue à Perec voir aussi KylouseK Petr (2015), Tarmac ou l'apocalypse selon Nicolas
} 
symbolique qui fournit au lecteur la clé pour l'interprétation de l'espace et du temps de Tarmac. Son importance consiste dans le rôle qu'il joue à la fois dans l'histoire et dans le récit. Il a été déjà mentionné que le terrain de baseball est le lieu de deux rencontres cruciales du roman. Il est également à noter que Hope à Rivière-du-Loup et Kamajii à Tokyo portent une casquette des Mets de New York, le club de Yogi Berra. De plus, la structure du roman peut être visualisée en tant que champ intérieur d'un terrain de baseball. L'incipit et l'excipit sont situés dans le marbre tout en constituant le point de départ et d'arrivée du jeu. Les chapitres 25, 49 et 73 représentent les trois bases, séparées du marbre ainsi que l'une de l'autre par une « distance » égale de 23 chapitres. Comme il a déjà été mentionné, Hope en tant que lanceur et Mickey en tant que receveur jouent dans la défense, ils luttent contre l'espace et contre le temps qui séparent non seulement les différentes bases du jeu, mais aussi la périphérie et le centre et, dans la seconde partie du roman, également Mickey et Hope. La métaphore du baseball actualise celle de la boucle. Si au niveau de l'histoire la boucle reste ouverte car le voyage de Mickey est amorcé sans être accompli, au niveau du récit elle est fermée. «Tout allait mieux depuis que la fin du monde était derrière nous. » (p. 259) Cette dernière phrase du roman réinstaure la question de l'avenir appartenant désormais au passé, c'est-à-dire la question inaugurée par l'épigraphe. Au moment où les héros décident d'en finir avec le passé (la tradition familiale), de cesser de redouter l'avenir (la fin du monde) et d'annuler la distance qui les sépare, une nouvelle période s'ouvre devant eux, la période où ils se rendent compte de l'importance de vivre ici et maintenant, de vivre tout simplement car jusque-là, ils ne faisaient que tuer le temps.

\section{BIBLIOGRAPHIE}

BERRA Yogi (1998), The Yogi Book: I really didn't say everything I said!, New York, Workman Publishing.

BILODEAU Anne-Marie (2015), Critique littéraire : Six degrés de liberté de Nicolas Dickner, L'Impact Campus, 16 mars 2015, en ligne : http://impactcampus.ca/arts-et-culture/ou-tes-papa-zoulou-ou-tes.

CAMERON Anne-Josée (2010), Nicolas Dickner : La fin du monde est en juillet, Revue Les libraires, 13 avril 2010, en ligne :

http://revue.leslibraires.ca/entrevues/litteraturequebecoise/nicolasdicknerlafind umondeestenjuillet.

CUSSON Chantale (2015), Raconte-moi un auteur : Nicolas Dickner, L'actualité, 28 oct 2015, en ligne :

http://www.lactualite.com/culture/livres/racontemoiunauteurnicolasdickner/.

DESLOGES Josianne (2013), Nicolas Dickner : fantasme postgéographique, Le Soleil, 19 octobre 2013, en ligne : http://www.lapresse.ca/lesoleil/arts/livres/201310/18/014700901nicolasdickne rfantasmepostgeographique.php. 
DeSMEules Christian (2015), «Six degrés de liberté » de Nicolas Dickner : un défi à la gravité, Le Devoir, 14 mars 2015, en ligne :

http://www.ledevoir.com/culture/livres/434328/six-degres-de-liberte-denicolas-dickner-un-defi-a-la-gravite.

KYLOUŠEK Petr (2015), Tarmac ou l'apocalypse selon Nicolas Dickner, in : DOLEŽALOVÁ Pavla, BAKEŠOVÁ Václava (eds.), La littérature qui fait respirer. Mélanges en l'honneur de Jiři Šrámek, Brno, MU, p. 29-42.

LAPOINTE Josée (2015), Nicolas Dickner : le roman du conteneur, La Presse, le 20 mars 2015, en ligne :

http://www.lapresse.ca/arts/livres/entrevues/201503/20/014853953nicolasdick nerleromanduconteneur.php.

OTIS Christine (2009), Le jeu des coïncidences : une vraisemblance à construire. Les exemples de Nikolski de Nicolas Dickner et de La kermesse de Daniel Poliquin, Temps zéro, $\mathrm{n}^{\circ} 2$, en ligne :

http://tempszero.contemporain.info/document398.

SIMARD-HOUDE Mélodie (2009), Réécrire Babel à l'ère médiatique, Salon double. Observatoire de la littérature contemporaine, en ligne :

http://salondouble.contemporain.info/lecture/reecrirebabelaleremediatique.

VALERY Paul (1945), Regard sur le monde actuel et autres essais, Paris, Gallimard. (2015) La bibliothèque idéale de Nicolas Dickner, La librairie Gallimard de Montréal, 23 avril 2015, en ligne :

https://www.gallimardmontreal.com/system/ckeditor_assets/attachments/143/a ffiche liste bibliotheque nicolas.pdf?1429822545. 